\title{
Signal Strength based Scanning Considering Free Capacity for Handover Execution in WiMAX Networks
}

\author{
P.P. Edwin Winston \\ Asst. Professor \\ Dept. of ETCE \\ Sathyabama University
}

\author{
K.S. Shaji, PhD. \\ Principal \\ RIITW
}

\begin{abstract}
In mobile networks handover is initiated when there is change in network Quality of Service (QoS) parameters such as signal strength, signal to interference noise ratio (SINR), bit error rate (BER) etc. Handover considering signal strength is a basic scheme in mobile networks. Considering only signal strength is not ideal for WiMAX networks, which has stringent QoS requirements. Signal strength along with hysteresis and threshold serves as an accurate handover mechanism in WiMAX network. But it is not necessary that selected target base station have enough capacity to handle the data of mobile station. In this paper we propose a multiple trigger initiated handover execution which considers the signal strength along with hysteresis and threshold during scanning phase and the estimation of free capacity during handover execution phase. We evaluate our proposed scheme in Opnet Modeler simulator and its performance is verified.
\end{abstract}

\section{Keywords}

Handover, WiMAX, Multiple trigger, OPNET.

\section{INTRODUCTION}

When a mobile station(MS) moves from one base station(BS) to another, the MS must be served by a new BS. This process of transferring connection of MS from one BS to another BS without interruption of service is called handover( $\mathrm{HO})$.

In mobile WiMAX the handover process can be divided into initiation phase and execution phase. During initiation phase the MS scans the NBSs for QoS parameters such as signal strength, Bit error rate(BER), signal to interference ratio, distance, velocity etc. Based on the scanning information the $\mathrm{HO}$ is executed. If the handover does not take place at the right time the ongoing call will be dropped [1],[2],[3].

Handover based on signal strength is more effective and simple. It can be classified into signal strength with hysteresis and signal strength with threshold. In signal strength with threshold the MS requests for a handover if the signal strength level from the serving base station falls below a threshold signal level. In signal strength with hysteresis the MS requests for a handover if the signal strength from the SBS falls below the signal level of the neighboring potential target base station by a fixed value called hysteresis margin.

The resource available on a wimax BS for data transmission to MS is explained in terms of effective capacity. The capacity of the interfaces involved can be used to estimate data rates in communication systems.

Our approach considers signal strength with hysteresis and threshold during scanning phase and the estimation of free capacity during handover execution phase. Performance of our approach can be determined based on number of unnecessary handovers, handover delay etc. We have analyzed the performance of our approach using opnet modeler network simulator.

\section{WIMAX HANDOVER IN VEHICULAR NETWORKS}

In mobile communications, handover is the process of transferring an ongoing connection from one base station to another base station depending on the QoS requirements of the mobile station.

Mobile WiMAX supports two types of handover at the link layer ie, inside a network. Hard handover is the default handover mechanism in a WiMAX network and soft handover is the optional handover mechanism. Hard handover is a Break-before-make procedure whereas soft handover is a Make-before-break procedure. Macro-diversity handover(MDHO) and Fast Base Station Switching(FBSS) are the soft handover mechanism in mobile WiMAX [4],[5]

Both these handovers follows the initial scanning phase and the final actual handover phase. During the scanning phase the MS scans the neighboring base station for finding the target base station. Once the target base station is selected the actual handover process is performed and MS is connected to the new target base station.

Vehicular communication is an emerging class of wireless communication enabling mobile users in their vehicles to communicate to the road and to each other. Numerous technologies have been deployed to assist and manage transportation over the years. But recent concerted efforts in academia and industry point to a paradigm shift in intelligent transportation systems.

Vehicles will carry computing and communication platforms and will have enhanced sensing capabilities. They will enable new versatile systems that enhance transportation safety and efficiency. A key component of Intelligent Transportation Systems (ITS) is the provision of adequate network infrastructure to support vehicular communication. Vehicular communication are expected to take place in urban zones, rural zones and highways through providing some network functionalities, protocols and integration strategies for services delivery to users. Consequently, vehicular networks are promising in providing a set of on-board potential services for drivers and passengers as well as providing different communication facilities between moving vehicles. A typical vehicular network is shown in figure 1 . 


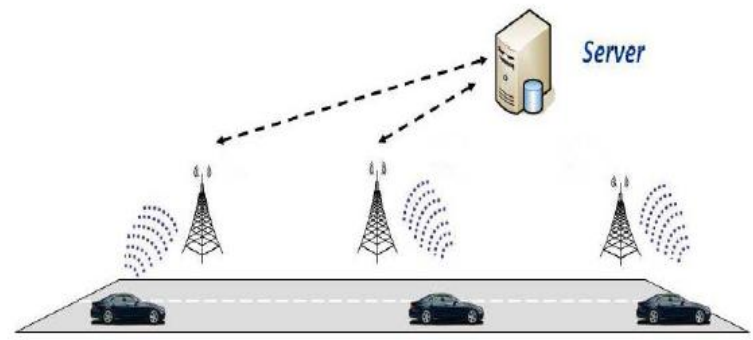

Fig. 1. Vehicular Network

Vehicular Networks create some challenges which can greatly impact the future deployment of these networks. Firstly, fast association and low communication latency should be satisfied between communicating vehicles in order to guarantee: i) service's reliability for safety-related applications while taking into consideration the time sensitivity during messages transfer, and ii) the quality and continuity of service for passenger's oriented applications.

The development of such vehicular networks and related technologies has been the subject of numerous projects around the globe, as well as for standardization working groups and industrial consortia. Applications in intelligent communication technologies for intelligent transport systems are one of the key means to ensure safe and efficient driving through the increasingly overloaded road infrastructure. The most recent development is the implementation of cooperative traffic management systems, with the establishment of a wireless infrastructure-to-vehicle (I2V) data-communication link for the transmission of safety critical as well as legally binding messages into the vehicle.

\section{SIGNAL STRENGTH BASED \\ HANDOVER}

The mobile station estimates the signal strengths from the neighboring BSs as shown in figure 2 and the value of the received signal level is the sum of three parameters namely path loss, shadow fading and multipath fading[6],[7],[8].

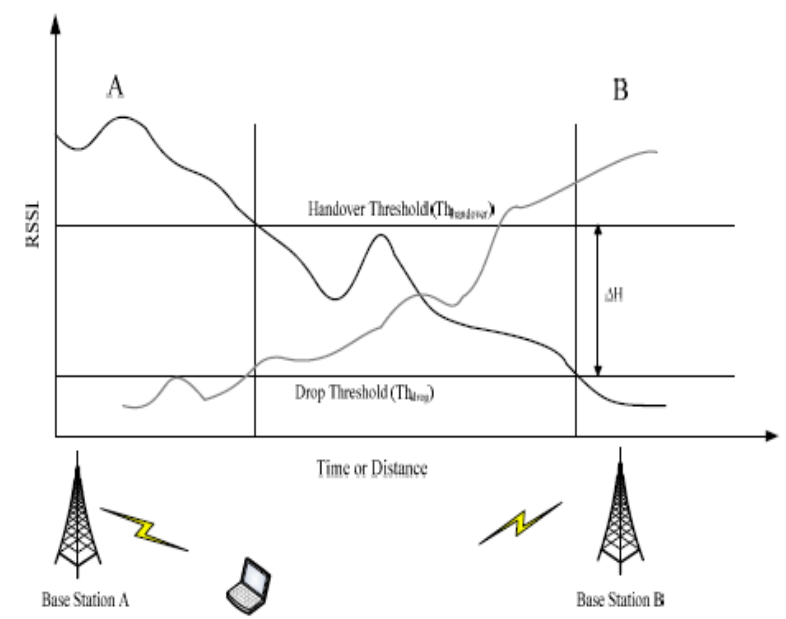

Fig. 2. Signal strength based Handover

Path loss depends on the distance of MS from a BS. It increases with the distance from BS. Between base station and mobile station, there are many obstacles. Those obstacles create variation of signal strength over the mean path loss. This variation is known as shadow fading which follows log normal distribution. Mobile station receives line of sight (LOS) signal from BS and also reflected signals. Those multipath components result multipath fading. Multipath fading is found to follow Rayleigh distribution[9],[10],[11].

\subsection{Handover considering signal strength}

If the received signal from $\mathrm{BS} 1$ is less than the received signal from BS2 then there is handover from BS1 to BS2. The MS selects the BS which provides higher signal strength. The decision is based on a mean measurement of the received signal. This method is observed to provoke too many unnecessary handovers, even when the signal of the current BS is still at an acceptable level.

\subsection{Handover considering signal strength with hysteresis}

If the received signal strength from BS1 is less than the received signal strength from BS2 by a margin ' $h$ ', then there will be handover from BS1 to BS2. Similarly if the signal strength of BS2 is less than the signal strength of BS1 by a margin ' $h$ ', then there will be handover from BS2 to BS1. In this method we cannot guarantee QoS requirements since both the signal strengths could be weaker. This technique prevents ping-pong effect.

\subsection{Handover considering signal strength with threshold}

When the received signal from BS1 is less than a specified value 'Pmin' and at the same time received signal from BS2 is more than Pmin, then for continuation of the call handover will take place from BS1 to BS2. Here Pmin is the minimum value of received power for which call is possible. If the signal strength drops less than Pmin, then there will be call drop for ongoing call and the new call will not be possible. If the received signal strengths from both the BSs go below Pmin, then call will be dropped and there will be outage. This results in additional interference to co-channel users. Thus , this scheme may create overlapping cell coverage areas. A threshold is not used alone in actual practice because its effectiveness depends on prior knowledge of the crossover signal strength between the current and candidate BSs.

\subsection{Handover considering signal strength with hysteresis and threshold}

This scheme hands a MS over to a new BS only if the current signal level drops below a threshold and the target BS is stronger than the current one by a given hysteresis margin.

\subsection{Handover considering free capacity}

The resource available on a wimax BS for data transmission to MS is explained in terms of effective capacity. The capacity of the interfaces involved can be used to estimate data rates in communication systems. Each BS can estimate its maximum effective capacity on a real-time basis. Therefore, each BS could obtain the effective idle capacity:

$$
\text { Cidle }=\text { Ceffective }- \text { Cthroughput }
$$

\section{PROPOSED MULTI TRIGGER HANDOVER}

Since the Handover latency is a main contributor in the percentage of packets lost, decreasing the number of $\mathrm{HO}$ will decrease the probability of packet loss. Furthermore, a wrong HO decision will lead to more HOs on the same path which in turn will increase the number of packets lost. Wrong HO 
decision can easily occur due to the harsh environment and its dynamics.

Our proposed scheme is a combination of existing handover mechanisms. It is a hybrid of MS initiated (signal strength with hysteresis and threshold) and BS initiated (capacity) handovers.

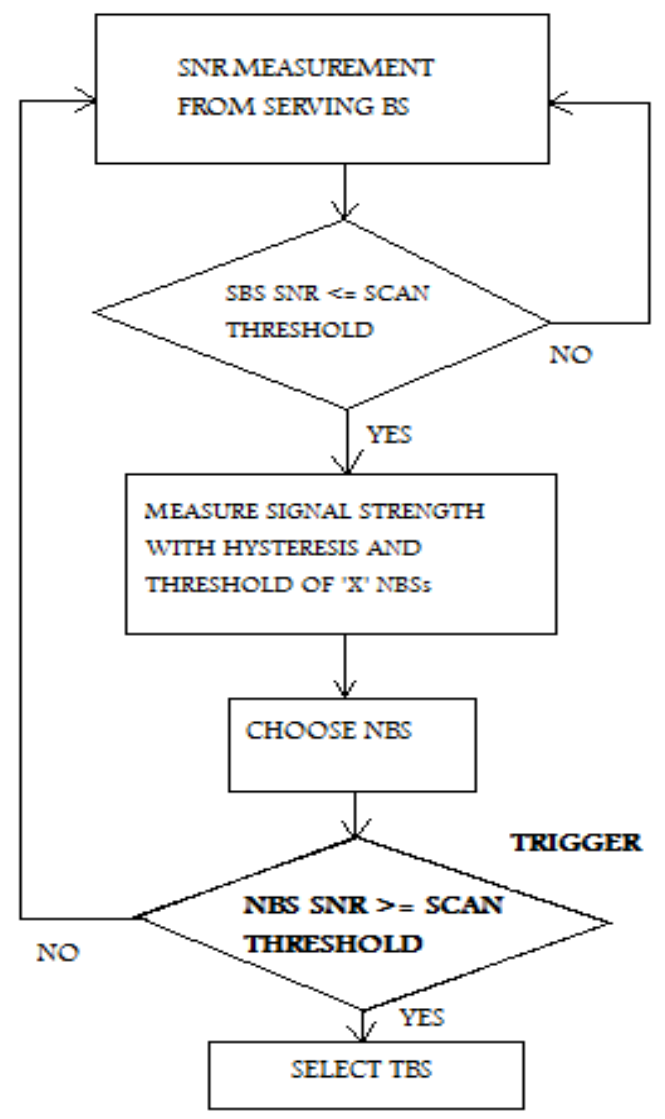

Fig. 3. Trigger during scanning phase

The proposed scheme prevents an MS from performing the handover if the target BS has no free capacity. Once the MS reaches the scanning threshold fixed by the signal strength with hysteresis and threshold algorithm, it sends MOB_SCN_REQ message to the serving BS and starts the scanning process. The MS uses these parameters to compute the QoS of the neighboring BSs and sends it in a MOB_MSHO_REQ message to the target BS during the handover process.

The proposed handover algorithm defines a new multi triggering mechanism based on computation of SNR during scanning phase and the estimation of free capacity during the handover execution phase. Figure 3 shows the triggering mechanism during the scanning phase. Figure 4 shows the triggering mechanism during the handover phase. The capacity limit is calculated in such a way that the current capacity must be greater than the maximum capacity times threshold hysteresis. This approach reduces the probability of call loss since no call will be dropped even if the BS is operating close to its capacity limit. The proposed triggering condition is defined as:

SNRmaxDT - SNRDS > H1

and
$\mathrm{CEF}>=\mathrm{H} 2 * \mathrm{Cmax}$

where SNRmaxDT - the maximum downlink SNR of the target BS

SNRDS - the downlink SNR of the serving BS

CEF - the estimated free capacity of the serving BS

Cmax-- the maximum free capacity of the serving BS

$\mathrm{H} 1$ and $\mathrm{H} 2$ - first and second handover threshold hysteresis

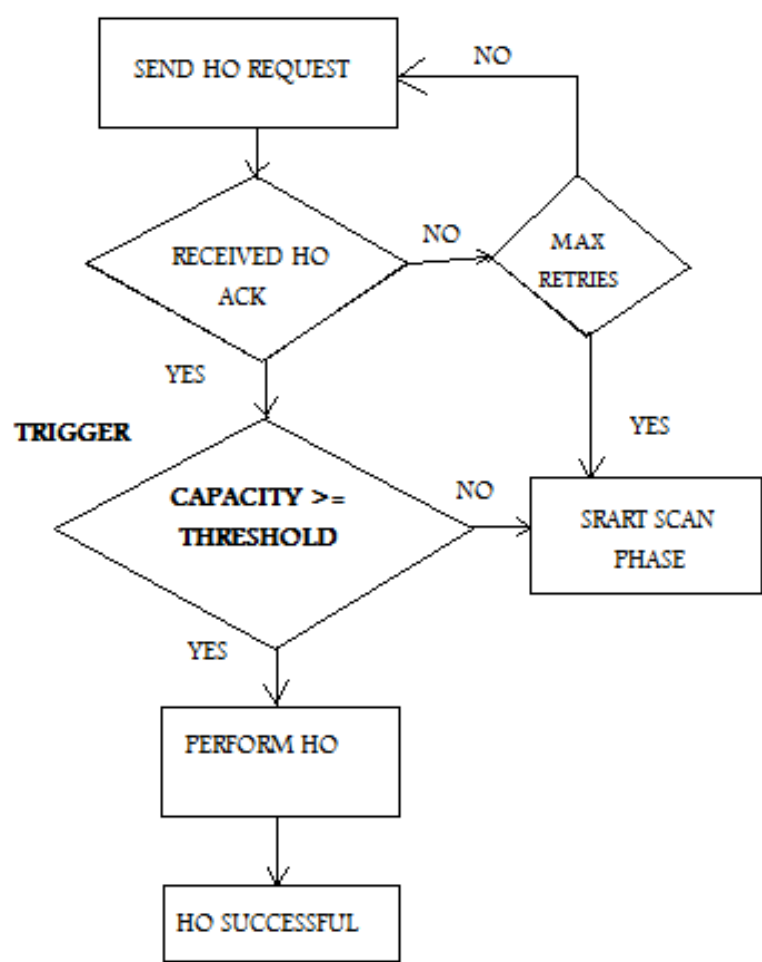

Fig. 4. Trigger during handover phase

During the scanning phase, if the signal strength with hysteresis and threshold of the NBS is greater than the SBS (serving base station), then scanning operation is triggered by selecting the target base station. During the handover execution phase, if the capacity of the target base station is greater than the capacity threshold, then handover execution operation is triggered by handing over to the target base station. Using multiple value triggers can be more efficient and will decrease the number of unwanted HOs which in turn decreases the probability of consecutive packet loss.

\section{NETWORK SETUP}

As shown in the architecture(Fig.5), there are six WiMAX base stations which are distributed all around the city. All the base stations are connected through wire to the correspondent node $(\mathrm{CN})$ which is similar to the server. The mobile node $(\mathrm{MN})$ which is nothing but a vehicle having a WiMAX mobile phone is connected to any one of the base stations and is moving around all the six base stations. It is connected wirelessly to the base stations.

This experiment is constructed in OPNET Modeler. In this paper two scenarios have been created. The first one uses only the SNR trigger, where the handover depends on signal 
strength with hysteresis and threshold. In the second one, in order to increase the throughput our proposed multiple trigger handover scheme is employed. Here the handover depend on signal strength with hysteresis and threshold and capacity. The I2V traffic information is broadcasted from the Correspondent Node $(\mathrm{CN})$ through the infrastructure to all the MN's. The simulation is performed at vehicular speeds.

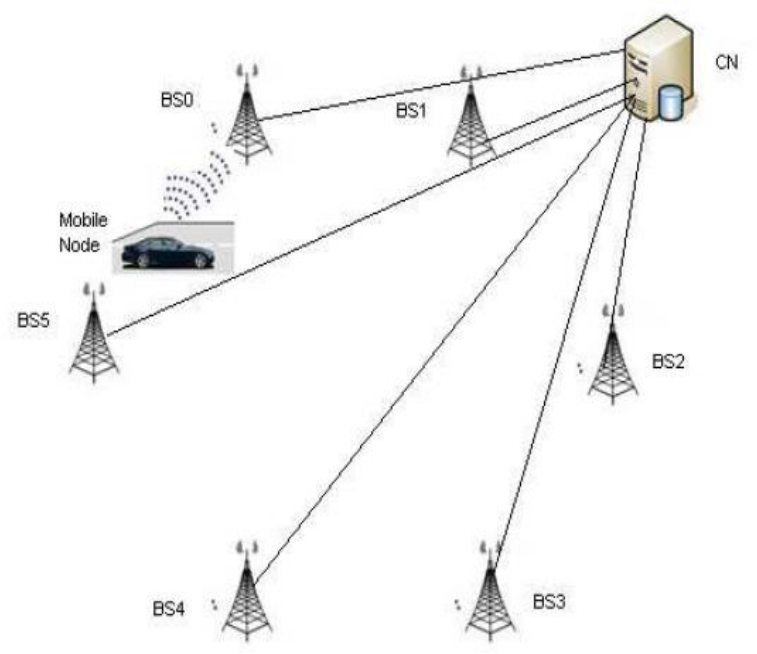

Fig. 5. Network Setup

\section{PERFORMANCE ANALYSIS}

The performance of the proposed scheme is evaluated using the Network simulator OPNET Modeler. The simulation was performed for various speeds according to vehicular environment. The performance graph shows an increase in the network throughput for our proposed scheme as shown in Fig.6.

Multiple Trigger Handover(MTH) improves performance by decreasing the unwanted $\mathrm{HO}$ from $8-10 \%$ to less than $2 \%$. MTH also eliminates the consecutive packet loss.

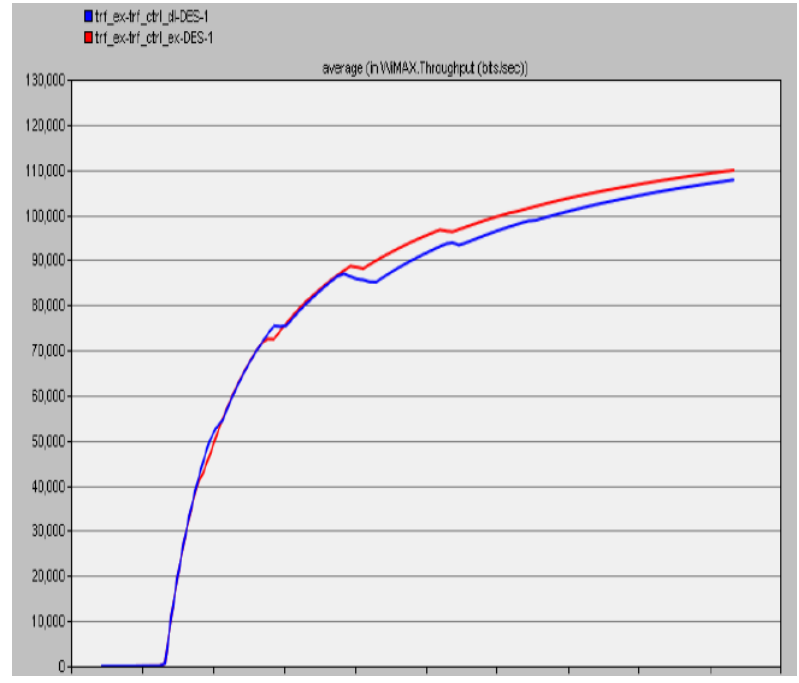

Fig. 6. Performance Graph

\section{CONCLUSION}

Since handover is a complex procedure, it is necessary to avoid unnecessary handovers. In this work we have considered multiple conditions in terms of signal strength and available capacity. Considering multiple conditions or triggers before handover execution greatly improves the handover performance in terms of throughput and delay. Though handover execution which considers multiple triggers is less complex inside a network, it faces lot of challenges in an internetwork. Proposing ideal triggering in internetwork handovers is surely a future research issue.

\section{REFERENCES}

[1] R. Vijayan and J. Holtzman, "A model for analyzing handoff algorithms," IEEE Transactions on Vehicular Technology, Vol. 42, No. 3, pp. 351-356, August 1993.

[2] G. E. Corazza, et al., "Characterization of handover initialization in cellular mobile radio networks," IEEE VTC' 94, pp. 1869-1872, 1994.

[3] T. S. Rappaport, "Wireless communications," Prentice Hall, 1996.

[4] D. Nisith, et al., "Handoff in cellular systems," IEEE Personal Communications, Dec. 1998.

[5] M. Gudmundson, "Analysis of Handover algorithm", IEEE VTC, May 1991, pp 537-42.

[6] M. Gudmundson, "Correlation model for shadow fading in mobile radio systems," Electronics Letters, Vol. 27, No. 23, pp. 2145-2146, November 1991.

[7] G. P. Pollini, "Trends in handover design," IEEE Communication Magazine, Vol. 34, pp. 82-90, March 1996.

[8] G. P. Pollini, "Handover rate in cellular systems: Towards a closed form approximation," Global Telecommunications Conference, GLOBE COM'97, IEEE, Vol. 1, pp. 711-715, November 1997.

[9] N. Zhang and J. M. Holtzman, "Analysis of handover algorithms using both absolute and relative measurements," IEEE VTC'94, pp. 82-86, 1994.

[10] B. Singh, et al., "Sensitivity analysis of handover performance to shadow fading in microcellular systems," IEEE ICPWC, 2005.

[11] S. D. Roy, "Effects of averaging, shadow fading and hysteresis margin on handover performance," CD proceedings, ICEMC, Pesit, Bangalore, Aug. 2007. 